Lengua \& Sociedad, Revista de Lingüística Teórica y Aplicada

Vol. 18, n. ${ }^{\circ}$ 2, Lima, julio-diciembre de 2019, pp. 123-138

https://doi.org/10.15381/lengsoc.v18i2.22332

\title{
Las denominaciones de los lugareños de Lima Metropolitana y el Callao: gentilicios y lexicografía
}

\section{The denominations of the locals of Metropolitan Lima and Callao: gentilics y lexicography}

\author{
Marco Antonio Lovón Cueva \\ Universidad Nacional Mayor de San Marcos, Lima, Perú \\ Grupo de Investigación Lexi \\ mlovonc@unmsm.edu.pe \\ https://orcid.org/oooo-0oo2-9182-6072 \\ Claudia Ximena Garay Ramírez \\ Universidad Nacional Mayor de San Marcos, Lima, Perú \\ Grupo de Investigación Lenguas y Filosofías del Perú \\ cgarayr@unmsm.edu.pe \\ https://orcid.org/oooo-0002-2999-8914
}

\begin{abstract}
Resumen
Los gentilicios se utilizan para referirse a las personas por su lugar de origen. En el Perú, son casi nulos los estudios que se centren en las denominaciones de los lugareños de una ciudad. El objetivo del presente artículo es analizar los gentilicios de los distritos de la provincia de Lima, aquellos que pertenecen a Lima Metropolitana y la Provincia Constitucional del Callao. Para esto, se enlista los vocablos y se estudian de acuerdo con la técnica lexicográfica. El trabajo concluye con la importancia de conocer los gentilicios, su significado, su escritura y sus variantes ortográficas, rasgos vinculados con la identidad de los lugareños.
\end{abstract}

Palabras claves: gentilicio, distrito, Lima, lexicología, lexicografía, sociedad.

\begin{abstract}
The gentilics are used to refer to the name given to the people from a particular region or country. In Peru, studies that focus on the denominations of the place residents are almost nil. The objective of this survey is to analyze the gentilics of the districts of the province of Lima, those that belong to Metropolitan Lima and the Constitutional Province of Callao. For this, the words are listed and studied according to the lexicographic technique. The research concludes with the importance of knowing the gentilics, their meaning, their writing and their spelling variants, features linked to the identity of the locals.
\end{abstract}

Key word: gentilic, district, Lima, lexicology, lexicography, society. 


\section{Introducción}

Hasta el año 1999, existían pocos estudios sobre los gentilicios o demónimos en español. Ello es una consecuencia de los pocos listados de los que se disponía. Algunos de estos han sido registrados en el diccionario ideológico de Casares, tratados de modo independiente, fuera del cuerpo de entradas lexicográficas, en un apéndice, y en el diccionario de Real Academia Española en su ortografía de aquel año, fundamentalmente de «nombres de países reconocidos por los organismos internacionales, con sus capitales y gentilicios» (RAE, 2014, apéndice 2). En la actualidad, este panorama no ha tenido un cambio drástico, si bien existen algunos artículos cuyo fin es recolectar los gentilicios de diversos países, distritos, departamentos, etc., aún no hay estudios específicos ni por el lado de la morfología ni por el lado de la lexicografía. En otras palabras, no son los suficientes.

En el Perú, son escasos los estudios de gentilicios, a lo mucho se restringen estudios de onomástico, antroponimia o etnonimia en la zona andina (CerrónPalomino, 2008, Lovón, 2019). Pero no hay estudios centrados en los gentilicios de los distritos de la metrópoli o de otras regiones del país. Los sistemas de administración, especialmente los de registro de municipios, no tienen siempre tampoco claro el gentilicio del lugar o de otros distritos, porque no existe aún un trabajo que recoja todos los casos de denominaciones de los ciudadanos peruanos de todos sus rincones. En este caso, es necesario realiza un primer estudio sobre los gentilicios de la capital, pues en Lima se encuentra la mayoría de peruanos, e incluso de extranjeros.

Consideramos importante el recojo de los gentilicios, en tanto se muestre el uso que hacen los hablantes de la lengua. En este trabajo, estudiamos los gentilicios, específicamente los gentilicios de los distritos de Lima Metropolitana.

En principio, se recolectan los gentilicios del departamento de Lima y la Provincia Constitucional del Callao, y, luego, se pasa a definir y ejemplificar cada una de las entradas léxicas.

\section{Los gentilicios}

El gentilicio es el adjetivo o sustantivo que expresa origen geográfico (RAE, 2014). Los gentilicios son adjetivos, en ocasiones también sustantivados (Bosque, 1997, p. 65), que derivan de nombres propios de lugar; en cuanto tales, su estudio compete a la deonomástica, en concreto, a la deonomástica toponímica. 
Las denominaciones de los lugareños de Lima Metropolitana y el Callao...

Los gentilicios designan principalmente a los oriundos y habitantes de diversos tipos de lugares (continentes, regiones, ciudades). También, comprenden las denominaciones de etnias, pueblos, nacionalidades, lenguas, etc. (Schweickard, 1992; García, 1997). Por ejemplo, el gentilicio del natural o habitante de Venezuela es venezolano (Lovón y Pita, 2016). De esta manera, los gentilicios se diferencian de los apodos o sobrenombres, que refieren a rasgos étnicos o personales vistos como defectos o particulares, que a rasgos de locación u origen (Lovón y Jiménez, 2016).

Los gentilicios también son conocidos como deonomásticos, e incluso como demónimos. Y son casos de deantroponimia, es decir, son usos deantroponímicos (García y Batista, 2018), o casos de onomosiología (Kordic \& Chávez, 2017).

García y Morera (2015) señalan que tenemos adjetivos gentilicios que expresan la relación de las personas, animales y cosas con el lugar que se trata de forma gramatical, mediante un complemento morfológico añadido al nombre propio de ese lugar. Es el caso de las formas peruano, habanero y francés, por ejemplo, derivados de los topónimos Perú, La Habana y Francia, mediante los sufijos -ano, -ero y -és, respectivamente. El vínculo con el lugar se expresa aquí de forma relativa: peruano se dice de la persona, animal o cosa que guarda algún tipo de relación (la que indica el sufijo -ano) con el lugar llamado Perú; habanero, de la persona, animal o cosa que guarda algún tipo de relación (la que indica el sufijo - ero) con el lugar llamado La Habana; y francés, de la persona, animal o cosa que guarda algún tipo de relación (la que indica el sufijo -és) con el lugar llamado Francia. De otra parte, tenemos adjetivos gentilicios que expresan la relación de las personas, animales y cosas con el lugar de que se trata de forma léxica, mediante un adjetivo primitivo o derivado que nada tiene que ver con el nombre de lugar implicado en el gentilicio referenciado, o que es anterior a él. Es lo que sucede en el caso de las formas hispano, italo, ruso, bereber, franco, moro o maqueto, por ejemplo. El vínculo con el lugar se expresa en este caso no de forma relativa, sino de forma absoluta: franco no es, en principio, el «natural de Francia», sino una persona que vive libre o no sometida al poder romano.

\section{Metodología}

Esta es una investigación cualitativa, descriptiva. En esta, se optó por organizar la información recogida presentándolo en forma de fichas lexicográficas. Dichas fichas presentan la siguiente estructura: entrada, marcas lingüísticas, definiciones y ejemplos de uso. Las entradas siguen un orden alfabético. En el caso de las 
definiciones, se procura recurrir a definiciones propias o perifrásticas, siguiendo de ejemplo a Porto Dapena (2002).

Los ejemplos de uso han sido recogidos de muestras brindadas por los habitantes de los distritos de Lima durante los meses de abril a junio del presente año. Estos son hablantes de castellano peruano. Se ha optado por este grupo porque, por un lado, atestigua la vigencia de la voz estudiada, y, por otro lado, porque permite recoger nominaciones en el habla coloquial y popular del Perú. En nuestra encuesta, formulamos dos preguntas: 1) ¿De qué distrito es usted? 2) ¿Cómo son llamados la gente de su distrito (Escriba un ejemplo como el siguiente: Soy de... Madrid... y nos llaman... madrileño...)? Contamos con 30 sujetos encuestados que los identificamos con los códigos i para señalar que se trata de la información obtenida por un informante y el número de encuesta del 1 al 30 con el que se le otorgó.

Luego, se optó por someter los ejemplos de uso, brindados por los informantes, a validación al buscar su empleo en diversas páginas de internet (Facebook, diarios web, blogs, páginas de las municipalidades, etc.). Cabe recalcar que los distritos considerados para el siguiente estudio son solo los distritos pertenecientes a Lima Metropolitana, y los distritos pertenecientes a la Provincia Constitucional del Callao. El nombre de estos distritos fue obtenido del artículo del INEI del 2014. Los casos que no se ilustran se deben a que ameritan mayor contrastación. Las abreviaturas que se emplean en este trabajo son las siguientes:

$\begin{array}{ll}\text { m. } & \text { masculino } \\ \text { f. } & \text { femenino } \\ \text { sust. } & \text { sustantivo } \\ \text { adj. } & \text { adjetivo } \\ \text { coloq. } & \text { coloquial } \\ \text { pop. } & \text { popular } \\ \text { consu. } & \text { consultado }\end{array}$

En las segundas acepciones, se coloca negrita a la remisión. 
Las denominaciones de los lugareños de Lima Metropolitana y el Callao...

\section{Análisis lexicográfico}

\subsection{Listado de gentilicios}

\begin{tabular}{ll|ll} 
Distrito & gentilicio & Distrito & gentilicio \\
Ancón & anconeño, & Puente Piedra & puentepedrino \\
& anconense & Rímac & rimence \\
Ate Vitarte & ateño & San Bartolo & sanbartolino \\
Barranco & barranquino & San Borja & sanborjino \\
Breña & breñense & San Isidro & sanisidrino \\
Carabayllo & carabayllano & San Juan de Lurigancho & luriganchino \\
Chaclacayo & chaclacayino & San Juan de Miraflores & sanjuanino \\
Chorillos & chorrillano & San Luis & sanluisiano \\
Cieneguilla & cieneguillence & San Martín de Porres & sanmartino; \\
Comas & comeño & & sanmartiniano \\
El Agustino & agustiniano & San Miguel & sanmiguelino \\
Independencia & independiente & Santa Anita & santanitense \\
Jesús María & jesusmariano & Santa María del Mar & santamariense \\
La Molina & molinense, & Santa Rosa & santarrosano \\
& molinero & Santiago de Surco & surcano \\
La Victoria & victoriano & Surquillo & surquillano \\
Lima & limeño & Villa el Salvador & villasalvadoreño \\
Lince & linceño & Villa María del Triunfo & villamariano \\
Los Olivos & olivense & Ventanilla & ventanillense \\
Lurín & lureño & Callao & chalaco \\
Magdalena del Mar & magdalense & Bellavista & bellavistiano \\
Miraflores & miraflorino & Carmen de la Legua & chalaco \\
Pachacamac & pachacameño & La Perla & chalaco \\
Pucusana & pucusaneño & La Punta & chalaco \\
Pueblo Libre & pueblerino & Mi Perú & chalaco \\
& & &
\end{tabular}

\subsection{Fichas lexicográficas}

\section{agustiniano, $-\boldsymbol{n a}$.}

1. adj. /m. y f. pop. Natural o residente del distrito El Agustino. Ejemplo: Gracias vecino agustiniano por tu apoyo en cada caminar, en cada movilización, en cada gesto de apoyo esto aún no acaba seguiremos firmes en nuestras ideas $y$ pensares para nuestro amado Agustino. Gavidia Alcalde 2018. 18-09-18. https:// bit.ly/2AKpIkZ. Consu.: 04-10-19. 
2. adj. Perteneciente o relativo al distrito de El Agustino o a los agustinianos. Ejemplo: El suelo agustiniano, está constituido básicamente por clastos y suelos residuales de relativa compacidad. I-1

anconeño, $-\tilde{\boldsymbol{n}} \boldsymbol{a}$.

1. adj. /m. y f. pop. Natural o residente del distrito Ancón. Ejemplo: Manifestó otro de los anconeños que se sumó a las manifestaciones. RPP Noticias 23-02-11 https://bit.ly/2LPlXkF. Consu.: 04-10-19.

2. adj. Perteneciente o relativo al distrito de Ancón o a los anconeños. Ejemplo: Y dime: ¿te gustó el frescor de la playa anconeña? Arce, E. o8-07-11 https://bit.ly/31Obrwy. Consu.: 04-10-19.

\section{anconense.}

adj. /m. y f. pop. Natural o residente del distrito Ancón. Ejemplo: [...] como lo recuerdan todos los anconenses que trabajaron en la Anglo. Zeballos, C. 2017. El análisis del potencial turístico de lobitos como destino de turismo deportivo acuático, Talara, 2017. (tesis de licenciamiento). UsmP, Lima.

adj. Perteneciente o relativo al distrito de Ancón o a los anconenses. Ejemplo: [...] en menor medida, y algunos familiares de antiguos anconenses. karinamHA. 02-05-15. https://bit.ly/35bacti. Consu.: 04-10-19. ateño, $-\tilde{n} a$.

1. adj. /m. y f. pop. Natural o residente del distrito Ate. Ejemplo: Cerca del 80 por ciento de los ateños se autoidentifica como indígena. García, A. 16-10-03. https://bit.ly/2M7yuP2. Consu.: 04-10-19.

2. adj. Perteneciente o relativo al distrito de Ate o a los ateños. Ejemplo: Llevando regalos a más de 15,00o madres ateñas con apoyo de las macro zonales. Municipalidad de ate 13-05-19. https://bit.ly/2HgyoU5. Consu.: 04-10-19.

\section{barranquino, $-\boldsymbol{n} \boldsymbol{a}$.}

1. adj. /m. y f. pop. Natural o residente del distrito Ate. Ejemplo: Muchos de los barranquinos deseamos se coloquen tachos de basura en cada esquina a fin de poder tirar las bolsitas con desechos de nuestros canes. Terán, A. 03-06-19. https://bit.ly/2Om9Ioe. Consu.: 04-10-19.

2. adj. Perteneciente o relativo al distrito de Barranco o a los barranquinos. Ejemplo: [...] que hace que este barrio desprenda una «magia barraquina». Cooperating Volunteers, pequeñas acciones para cambiar el mundo. https:// bit.ly/31PJjlS. Consu.: 04-10-19. 
Las denominaciones de los lugareños de Lima Metropolitana y el Callao...

\section{bellavistiano, $-n a$.}

1. adj. /m. y f. pop. Natural o residente del distrito Bellavista. Ejemplo: Escuché hablar del candidato a regidor en Integración Bellavistiana. I-2

2. adj. Perteneciente o relativo al distrito de Bellavista o a los bellavistiano. Ejemplo: Los adolecentes bellavistianos escucharon la conferencia. Trujillo Di 25-05-11 https://bit.ly/334esci. Consu.: 04-10-19.

\section{breñense.}

1. adj. /m. y f. pop. Natural o residente del distrito Breña. Ejemplo: Este joven breñense, que cursa actualmente el Profesorado de Matemáticas. CharataChaco. net 01-05-19 https://bit.ly/2AH8ULB. Consu.: 04-10-19.

2. adj. Perteneciente o relativo al distrito de Breña o a los breñenses. Ejemplo: Clásico breñense en beneficio de los inundados. Sociedad. 31-01-19. https://bit. ly/2VdkDeb. Consu.: 04-10-19.

carabayllano, $-n a$.

1. adj. /m. y f. pop. Natural o residente del distrito Carabayllo. Ejemplo: ¡Vecino carabayllano! Te Invitamos a que revises el boletín informativo «Tu Muni», correspondiente al mes de setiembre. Municipalidad de Carabayllo. 03-10-17. https://bit.ly/2pw75Pm. Consu.: 04-10-19.

2. adj. Perteneciente o relativo al distrito de Carabayllo o a los carabayllanos. Ejemplo: Los parques carabayllanos están muy descuidados. I-3. chaclacayino, $-\boldsymbol{a}$.

1. adj. /m. y f. pop. Natural o residente del distrito Chaclacayo. Ejemplo: Los chaclacayinos en general les prestaron una primera y curiosa [...] para exigirle que tomara acción contra «la peste neonazi» que había [...]. Luis Freire. 2018. https://bit.ly/31Lgelb. Consu.: 04-10-19.

2. adj. Perteneciente o relativo al distrito de Chaclacayo o a los chaclacayinos. Ejemplo: En las vacaciones me voy a Chosica, me gustan los restaurantes chaclacayinos. I-4.

chorrillano, $-a$.

1. adj. /m. y f. pop. Natural o residente del distrito Chorrillos. Ejemplo: José Olaya fue un pescador chorrillano que entregó su vida por el Perú y que figura en los libros de historia. Peru21. 29-06-17 https://bit.ly/2FGOuI5. Consu.: 04-10-19.

2. adj. Perteneciente o relativo al distrito de Chorrillos o a los chorrilanos. Ejemplo: Agenda chorrillana. Municipalidad de Chorrillos. 03-10-19. https:// bit.ly/2Llyanp. Consu.: 04-10-19. 


\section{cieneguillence.}

1. adj. /m. y f. pop. Natural o residente del distrito Cieneguilla. Ejemplo: Conozco de una página de Facebook llamada el cieneguillance, porque muestra memes de lo sucedido en el distrito. I-5

2. adj. Perteneciente o relativo al distrito de Cieneguilla o a los cieneguillences. Ejemplo: Aclimatación cieneguillence. El Intérprete. 13-01-17. https:// bit.ly/2LK8PNB. Consu.: 04-10-19.

comeño, $-\tilde{n} a$.

1. adj. /m. y f. pop. Natural o residente del distrito Comas. Ejemplo: joven comeño, aprende y diviértete con nosotros en nuestro taller de batucada. Municipalidad Distrital de Comas. 22-05-19. https://bit.ly/357rebQ. Consu.: 04-10-19.

2. adj. Perteneciente o relativo al distrito de Comas o a los comeños. Ejemplo: Miguel Saldaña entrega credenciales a dirigentes y realiza mejoras al Parque del Niño Comeño. La Noticia. 02-06-16. https://bit.ly/3oSfiau. Consu.: 04-10-19. jesusmariano, $-n a$.

1. adj./m. y f. pop. Natural o residente del distrito Jesús María. Ejemplo: $Y$ más que eso, queremos hacer que nuestra gestión en la alcaldía sea motivadora de unión entre todos los jesusmarianos. Municipalidad de Jesús María. https:// bit.ly/2M9xkTm. Consu.: 04-10-19.

2. adj. Perteneciente o relativo al distrito de Jesús María o a los jesusmarianos. Ejemplo: Recorre los parques y plazas jesusmarianas. Al día con JM. https://bit.ly/30nxuAa. Consu.: 04-10-19.

jesusmariense.

adj. /m. y f. pop. Natural o residente del distrito Jesús María. Ejemplo: Jamás un jesusmariense estuvo con la expectativa de ser diputado nacional. Ariel Roggio 26-06-17. https://bit.ly/35oclLg. Consu.: 04-10-19.

adj. Perteneciente o relativo al distrito de Jesús María o a los jesusmarienses. Ejemplo: Los parques jesusmarienses son muy atractivos. I-12.

\section{limeño, $-\tilde{n} a$.}

1. adj. /m. y f. pop. Natural o residente del distrito Lima. Ejemplo: Los limeños suelen trabajar muchas horas. I-30

2. adj. Perteneciente o relativo al distrito de Lima o a los limeños. Ejemplo: [...] hoteles limeños favoritos para disfrutar la estadía en nuestra capital. Portal de Turismo. 23-08-19. https://bit.ly/2LLJItB. Consu.: 04-10-19. 
Las denominaciones de los lugareños de Lima Metropolitana y el Callao...

linceño, - $\tilde{\boldsymbol{n}} \boldsymbol{a}$.

1. adj. $/ \mathrm{m}$. y f. pop. Natural o residente del distrito Lince. Ejemplo: La Municipalidad de Lince sorteará un flamante auto o $\mathrm{kms}$ para los Vecinos Linceños Preferentes. Lince, respira limpio. 18-04-18. https://bit.ly/2LPfPsJ. Consu.: 04-10-19.

2. adj. Perteneciente o relativo al distrito de Lince o a los linceños. Ejemplo: [...] de brindar salud y bienestar a las mascotas de cada hogar linceño. Lince, respira limpio. 03-11-18. https://bit.ly/357M4L5. Consu.: 04-10-19.

lureño, $-\tilde{n} a$.

1. adj. /m. y f. pop. Natural o residente del distrito Lurín. Ejemplo: Yo soy lureña de corazón. I-6.

2. adj. Perteneciente o relativo al distrito de Lurín o a los lureños. Ejemplo: Mi mejor amiga vive cerca al distrito de Lurín, en una de las casas lureñas. I-7. molinense.

1. adj. /m. y f. pop. Natural o residente del distrito La Molina. Ejemplo: Los molinenses retiraron los avisos publicitarios. I-16

2. adj. Perteneciente o relativo al distrito de La Molina o a los molinenses. Ejemplo: Voluntariado molinense en acción. Municipalidad de la Molina 28-0516. https://bit.ly/31Ln2PL. Consu.: 04-10-19.

\section{magdalenense.}

1. adj./m. y f. pop. Natural o residente del distrito Magdalena del Mar. Ejemplo: Magdalenense de corazón, esposo y padre de familia. Municipalidad de Magdalena del Mar. https://bit.ly/2MfsuUz. Consu.: 04-10-19.

2. adj. Perteneciente o relativo al distrito de Magdalena o a los magdalenenses. Ejemplo: Promover el bienestar y la mejora de todos los vecinos magdalenenses. Plan de Gobierno Municipal Distrito de Magdalena del Mar 2019 2023. https://bit.ly/3oL7iuZ. Consu.: 04-10-19.

\section{miraflorino, $n a$.}

1. adj. /m. y f. pop. Natural o residente del distrito Miraflores. Ejemplo: Las miraflorinas son muy pitucas. I-17

2. adj. Perteneciente o relativo al distrito de Miraflores o a los miraflorinos. Ejemplo: También entablarán demanda de inconstitucionalidad contra la disposición del municipio miraflorino. Correo. 27-02-19. https://bit.ly/2pMeCtw. Consu.: 04-10-19. 
victoriano, $-n a$.

1. adj. /m. y f. pop. Natural o residente del distrito La Victoria. Ejemplo: Los victorianos son de Alianza Lima. I-4

2. adj. Perteneciente o relativo al distrito de La Victoria o a los victorianos. Ejemplo: los 75 parques victorianos, además de convertir los follajes y otros residuos [...] Andina, agencia peruana de noticias. 22-04-19. https://bit.ly/2OphzdP. Consu.: 04-10-19.

olivense.

1. adj. /m. y f. pop. Natural o residente del distrito Los Olivos. Ejemplo: Los olivenses son muy desordenados, por eso los inspectores son necesarios. I-8.

2. adj. Perteneciente o relativo al distrito de Los Olivos o a los olivenses. Ejemplo: [...] nuestro equipo de olivenses en acción en coordinación con nuestros vecinos iniciará los trabajos para cambiarle el rostro a nuestra comunidad. Olivenses en acción 18-08-17. https://bit.ly/2ALzT8V. Consu.: 04-10-19.

pachacameños, - $\tilde{n} a s$.

1. adj. /m. y f. pop. Natural o residente del distrito de Pachacamac. Ejemplo: Se suman los pachacameños al proyecto. I-9

2. adj. Perteneciente o relativo al distrito de Pachacamac o a los pachacameños. Ejemplo: Pero, significa que los sacerdotes pachacameños negociaron con los Incas. Perú y el grado de inversión Camu camu valiosa alternativa Pachacamac del Perú su Roma La patriótica Causa. https://bit.ly/2Om89Qj. Consu.: 04-10-19.

\section{pueblerino, $n a$.}

1. adj. /m. y f. pop. Natural o residente del distrito Pueblo Libre. Ejemplo: [...] Stephen Hass, quienes están celebrando los 197 años de fundación de ese histórico distrito. !Un abrazo a todos los pueblerinos! Acción Popular. 10-04-19. https:// bit.ly/2MkdIvI. Consu.: 04-10-19.

2. adj. Perteneciente o relativo al distrito de Pueblo Libre o a los pueblerinos. Ejemplo: Serenazgo de Pueblo Libre se dejó esperar casi dos horas a pesar de las [...] La pelea se dio entre un ex vecino del barrio pueblerino y el [...]. Sara Denegri Flores. 26-04-11. https://bit.ly/2Ir1fFo. Consu.: 04-10-19.

puentepedrino, $-n a$.

1. adj. /m. y f. pop. Natural o residente del distrito Puente Piedra. Ejemplo: Insólito: Puentepedrino no es discriminado en discoteca Aura. Deslengua2 Callao 15-11-14. https://bit.ly/33asrxz. Consu.: 04-10-19. 
Las denominaciones de los lugareños de Lima Metropolitana y el Callao...

2. adj. Perteneciente o relativo al distrito de Puente Piedra o a los puentepedrinos. Ejemplo: El 'Pisco puentepedrino' se lanzará el 14 de febrero con motivo de los 81 años de creación política de Puente Piedra. La República. 09-02-08. https://bit.ly/2AOZvYF. Consu.: 04-10-19.

pucusaneño, $-\tilde{n} a$.

1. adj. /m. y f. pop. Natural o residente del distrito Pucusana. Ejemplo: Mis familiares viven en Pucusana y se reconocen como pucusaneños. I-o9.

2. adj. Perteneciente o relativo al distrito de Pucusana o a los pucusaneños. Ejemplo: Vecinos de Pucusana alistan preparativos para el festival «Pucusaneño emprendedor». I-29.

\section{rimense.}

1. adj. /m. y f. pop. Natural o residente del distrito Rímac. Ejemplo: Vecinos rimenses, no te olvides que mañana jueves 21 se llevará a cabo la charla de sensibilización de canes en la Unidad vecinal del [...]. Municipalidad del Rímac oficial. 20-05-18. https://bit.ly/2MhkUsy. Consu.: 04-10-19.

2. adj. Perteneciente o relativo al distrito del Rímac o a los rimenses. Ejemplo: [...] lunes pasado, luego de su anuncio como nuevo DT del cuadro rimense [...]. Depor 06-02-19. https://bit.ly/2ouglaM. Consu.: 04-10-19.

\section{santamarinense.}

1. adj. /m. y f. pop. Natural o residente del distrito Santa María del Mar. Ejemplo: Los santamarinenses somos trabajadores, y luchamos por nuestros derechos laborales. I-11.

2. adj. Perteneciente o relativo al distrito de Santa María o a los santamarinenses. Ejemplo: Bellas mujeres santamarinenses participaron en el concurso. I-12.

\section{sanbartolino, $-\boldsymbol{n a}$.}

1. adj. /m. y f. pop. Natural o residente del distrito San Bartolo. Ejemplo: Vecino sanbartolino, aprovecha la amnistía tributaria y [...]. Municipalidad Distrital de San Bartolo. 27-02-19. https://bit.ly/2OsuOul. Consu.: 04-10-19.

2. adj. Perteneciente o relativo al distrito de San Bartolo o a los sanbartolinos. Ejemplo: Calidad de vida y desarrollo sostenible de los sanbartolinos contribuyendo [...]. Enciso Rufino. Plan de Gobierno San Bartolo. https://bit.ly/2ALhwxu. Consu.: 04-10-19. 
sanborjino, $-\boldsymbol{n a}$.

1. adj. /m. y f. pop. Natural o residente del distrito San Borja. Ejemplo: ¡Atención vecinos sanborjinos! Municipalidad De San Borja 22-11-17. https:// bit.ly/331N15R. Consu.: 04-10-19.

2. adj. Perteneciente o relativo al distrito del San Borja o a los sanborjinos. Ejemplo: ¿Cómo se encuentra hoy el museo sanborjino? Las autoridades respectivas indican que se invertirá 4 millones de soles en la remodelación [...]. El Comercio. 20-02-10. https://bit.ly/2oU98fA. Consu.: 04-10-19.

sanisidrino, $-n a$.

1. adj. /m. y f. pop. Natural o residente del distrito San Isidro. Ejemplo: Convocó a los ex alcaldes de San Isidro así como a sanisidrinos «notables». Félix Puémape. Dinámicas de poder a nivel local: el caso del distrito de san isidro (Lima, Perú) durante el periodo 2002-2014. https://bit.ly/2nkrZjN. Consu.: 04-10-19.

2. adj. Perteneciente o relativo al distrito de San Isidro o a los sanisidrinos. Ejemplo: [...] en sus conferencias serán alojados en este tradicional hotel sanisidrino. El Comercio. 08-06-18. https://bit.ly/2LPGwom. Consu.: 04-10-19.

\section{sanluisiano, $-\boldsymbol{n a}$.}

1. adj. /m. y f. pop. Natural o residente del distrito San Luis. Ejemplo: [...] quien desde su modesto despacho niega que los sanluisianos pertenezcan a [...]. El Tiempo. 21-10-96. https://bit.ly/336IQpr. Consu.: 04-10-19.

2. adj. Perteneciente o relativo al distrito de San Luis o a los sanlusianos. Ejemplo: Es una empresa sanluisiana de servicios públicos. I-13. santarrosano, - na.

1. adj. /m. y f. pop. Natural o residente del distrito Santa Rosa. Ejemplo: medalla al mérito otorgada al joven santarrosano. Grupo bosques y biodiversidad. 2018. https://bit.ly/2In5D8E. Consu.: 04-10-19.

2. adj. Perteneciente o relativo al distrito de Santa Rosa o a los santarrosanos. Ejemplo: En el tradicional chorizo santarrosano se emplean las mejores carnes del cerdo, picadas a mano, y se agregan cebolla, tomate, ajo, pimienta, comino $y$ otros $[. .$.$] . I-14.$

luriganchino, $-n a$.

1. adj. /m. y f. pop. Natural o residente del distrito San Juan de Lurigancho. Ejemplo: La cantante vernacular es luriganchina. I-9 
Las denominaciones de los lugareños de Lima Metropolitana y el Callao...

2. adj. Perteneciente o relativo al distrito de San Juan de Lurigancho o a los luriganchinos. Ejemplo: Roséll, cantautor luriganchino, presenta Momentos. SJL.pe 21-11-18. https://bit.ly/3516y8e. Consu.: 04-10-19.

sanjuanino, $-n a$.

1. adj. /m. y f. pop. Natural o residente del distrito San Juan. Ejemplo: El sanjuanino teme ser asaltado en el barrio la estación, La cueva del chancho o en cualquier lugar de Las Cheembas y Shawson. Acción Patética San Juan 26-o816. https://bit.ly/2Ip1jFD. Consu.: 04-10-19.

2. adj. Perteneciente o relativo al distrito de San Juan o a los sanjuaninos. Ejemplo: Señala que la mayoría de los parques en el distrito sanjuanino se encuentran descuidados. I-15.

sanmartino, $-\boldsymbol{n a}$.

1. adj. /m. y f. pop. Natural o residente del distrito de San Martín de Porres. Ejemplo: Sanmartiniana prioriza el proceso de elaboración del trabajo académico. León, V. 2018. Comunidades profesionales de aprendizaje para mejorar procesos didácticos en la resolución de problemas matemáticos de los estudiantes de educación primaria. Lima.

2. Perteneciente o relativo al distrito de San Martín de Porres o a los sanmartinianos. Ejemplo: [...] cultural y las capacidades comunicativas de los niños y niñas sanmartinianos. FODEP. https://bit.ly/20wVI77. Consu.: 04-10-19.

\section{sanmartinense.}

adj. /m. y f. pop. Natural o residente del distrito de San Martín de Porres. Ejemplo: Los sanmartinenses decidirán el destino político de nuestro país. I-11 adj. Perteneciente o relativo al distrito de San Martín de Porres o a los sanmartinenses. Ejemplo: Las empresas sanmartinenses solo invierten en hoteles. I-16.

\section{sanmiguelino, $-n a$.}

1. adj. /m. y f. pop. Natural o residente del distrito San Miguel. Ejemplo: Sanmiguelinos te esperamos en el cruce de las avenidas Precursores e Insurgentes. I-13

2. adj. Perteneciente o relativo al distrito de San Miguel o a los sanmiguelinos. Ejemplo: [...] y territoriales del distrito sanmiguelino se movilizaron por las calles [...]. ANRed. 25-10-17. https://bit.ly/2Oiuyzv. Consu.: 04-10-19. santanitense.

1. adj. /m. y f. pop. Natural o residente del distrito de Santa Anita. Ejemplo: Los santanitenses son muy buenos vecinos. I-14 
2. adj. Perteneciente o relativo al distrito de Santa Anita o a los santanitenses. Ejemplo: Banderazo santanitense. Jóvenes en Acción. 14-05-18. https:// bit.ly/2OmMaZy. Consu.: 04-10-19.

surcano, $-n a$.

1. adj. /m. y f. pop. Natural o residente del distrito de Surco. Ejemplo: Los surcanos denuncian robos. I-6

2. adj. Perteneciente o relativo al distrito de Surco o a los surcanos. Ejemplo: Reconozco las bellas piletas surcanas. I-17. surquillano, -na.

1. adj. /m. y f. pop. Natural o residente del distrito de Surquillo. Ejemplo: Reconocieron a surquillano ejemplar. I-3

2. adj. Perteneciente o relativo al distrito del Surquillo o a los surquillanos. Ejemplo: Vecino surquillano compartamos esta información y no se dejen sorprender. Municipalidad de Surquillo. 14-06-16. https://bit.ly/2LO5YTw. Consu.: 04-10-19.

villasalvadoreño, $\tilde{n} a$.

1. adj. /m. y f. pop. Natural o residente del distrito de Villa el Salvador. Ejemplo: ¡Invitación a todos los villasalvadoreños! Acción Popular-Villa el Salvador. 20-02-19. https://bit.ly/2pFAB58. Consu.: 04-10-19.

2. adj. Perteneciente o relativo al distrito de Villa el Salvador o a los villasalvadoreños. Ejemplo: Gerente de la empresa Austral afirmó que su empresa tiene el permiso de la comuna villasalvadoreña para laborar en el [...]. Radio Sorteo Villa. 22-11-17. https://bit.ly/339sJHL. Consu.: 04-10-19. villamariano, $-\boldsymbol{n a}$.

1. adj. /m. y f. pop. Natural o residente del distrito de Villa María del Triunfo. Ejemplo: Los parques y jardines son para los villamarianos. Municipalidad de Villa María del Triunfo. 14-03-19. https://bit.ly/30Q3LLM. Consu.: 04-10-19

2. adj. Perteneciente o relativo al distrito de Villa María del Triunfo o a los villamarianos. Ejemplo: El municipio villamariano 'amaneció' cerrado con candado y [...] Sabel Medina 10-10-18. https://bit.ly/30NwcoM. Consu.: 04-10-19. ventanillense.

1. adj. /m. y f. pop. Natural o residente del distrito de Ventanilla. Ejemplo: Los ventanillenses premiaron a los vecinos que cumplen con sus tributos. I-10

2. adj. Perteneciente o relativo al distrito del Ventanilla o a los ventanillenses. Ejemplo: acción cívica regional para pobladores de seis asentamientos humanos ventanillenses. Lamula.pe. 14-10-14. https://bit.ly/2Ina6rI. Consu.: 04-10-19. 
Las denominaciones de los lugareños de Lima Metropolitana y el Callao...

\section{Conclusiones}

En esta investigación lexicográfica, hemos compilado, ordenado, definido y ejemplificado los gentilicios sobre los lugareños de Lima Metropolitana y el Callao.

Como se ha podido observar, el listado está compuesto por sustantivos y adjetivos que refieren al origen geográfico en el que se localiza los ciudadanos limeños y todo lo concerniente o relacionado con su ciudad: Las limeñas son simpáticas, Yo soy limeño, Es un producto limeño, ¡Qué rico suspiro limeño!, son algunas maneras de usarlos. Los gentilicios o demónimos ponen de relevancia rasgos de identidad. Es decir, sirven para identificar la existencia de grupos sociales cohesionados, unidos por patrones comunes de residencia, o inclusive por normas culturales compartidas. En el habla de los ciudadanos, se puede encontrar ejemplos como Ojo, soy miraflorino, no sanisidrino, como forma de distinción. Trabajos posteriores podrán concentrarse en el estudio identitarios de los gentilicios. Con esta investigación, también se deja por sentado la ortografía de los gentilicios. Generalmente, los ciudadanos y los municipios locales tienen dudas de las denominaciones de sus vecinos o de su escritura.

Para finalizar, consideramos importante recopilar estos datos que se emplean en el léxico común del Perú, dado que son una muestra más del acervo lingüístico de los peruanos. Los gentilicios muchas veces no son estudiados. Por eso, este artículo es un acercamiento e invitación a estudios posteriores. Al respecto, sería interesante confeccionar un glosario o un diccionario de los gentilicios del Perú a nivel distrital y provincial. Se requiere examinar de qué manera operan dentro sus propios entornos culturales y distinguir los significados que adquieren.

\section{Referencias bibliográficas}

Academia Peruana de la Lengua (2016). DiPerú: diccionario de peruanismos. Lima: Compañía de Minas Buenaventura y Academia Peruana de la Lengua.

Bosque, I. (1999). El nombre común. Bosque, I. \& Demonte, V. (dirs.). Gramática descriptiva de la lengua española (p. 3-75). Madrid.

Cerrón-Palomino, R. (2008). Voces del Ande: Ensayos de onomástica andina. Lima: Pontificia Universidad Católica del Perú.

García, D. \& Batista, J. (2018). Adjetivos deantroponímicos con el sufijo -ano en español. Estudios de Lingüística del Español, (39), pp. 161-179. 
García, C. (1997). Deonomástica hispánica: vocabulario científico, humanístico y jergal. Madrid: Ed. Complutense.

García, D. \& Morera, M. (2015). Gentilicios y lexicografía. Onomazein: Revista semestral de lingüística, filología y traducción, (31), pp.81-98.

Hortúa, C. (2016). Diccionario de expresiones y léxico coloquial en Bogotá (Trabajo de Grado en Modalidad Creación e Interpretación Diccionario por Contextos Situacionales de Expresiones Idiomáticas y Léxico Coloquial en Bogotá). Bogotá, Universidad Distrital Francisco José de Caldas.

INEI. (2014). Una mirada a Lima metropolitana. Recuperado de: https:// www.inei.gob.pe/media/MenuRecursivo/publicaciones_digitales/Est/Lib1168/ libro.pdf

Kordic, R. \& Chávez, S. (2017). Qué se entiende por gentilicio. Aproximaciones onomasiológicas, sintácticas y morfológicas. Boletín de Filología, (1), 213-244.

Lovón, M. (2019). Las palabras compuestas en el aimara de Conima. Lexis, 53(1), pp. 55-85. https://doi.org/10.1880o/lexis.201901.002

Lovón, M. \& Jimenez, P. (2016). Apelativos futbolísticos en el Perú. Escritura y Pensamiento, 19(39), 191-2012. Recuperado de https://revistasinvestigacion. unmsm.edu.pe/index.php/letras/article/view/13724

Lovón, M. \& Pita, P. (2016). Los términos de la crisis venezolana. Boletín de Lingüística, 28(45-46), 79-110. Recuperado de http://190.169.94.12/ojs/index. php/rev_bl/article/view/14788

Núñez, Luis. (2005). La morfología derivativa en los gentilicios del español. Interlingüística, (15), pp. 1047-1056.

Schweickard, W. (1992). Deonomastik. Ableitungen auf der Basis von Eigennamen im Französischen (unter vergleichender Berücksichtigung des Italienischen, Rumänischen und Spanischen). Tübingen.

Pulido, D. \& Méndez, S. (2015). Léxico utilizado por los trabajadores de la pesca artesanal de lago y mar en las comunidades de la isla de Ometepe, isletas de Granada, playas de Casares y Salinas Grandes durante el segundo semestre de 2014. Managua, Universidad Nacional Autónoma de Nicaragua.

Real Academia Española (2014). Diccionario de la lengua española. Barcelona: Espasa. 